“ (C) 2016 IEEE. Personal use of this material is permitted. Permission from IEEE must be obtained for all other uses, in any current or future media, including

reprinting/republishing this material for advertising or promotional purposes, creating new collective works, for resale or redistribution to servers or lists, or reuse of any copyrighted component of this work in other works." 


\title{
Optimal Charging Strategy of Electric Vehicles Customers in a Smart Electrical Car Park
}

\author{
Tingting He*, Yingjie Bai ${ }^{\dagger}$, Jianguo Zhu* \\ *Faculty of Engineering and Information Technology, University of Technology Sydney, Sydney, Australia, \\ + Beijing National Railway Research \& Design Institute of Signal \&Communication Ltd, Beijing, China \\ Tingting.He@student.uts.edu.au, yingjiebai009@gmail.com and Jianguo.Zhu@uts.edu.au
}

Keywords: Electric vehicles, Smart electrical car park, Optimal charging strategy.

\begin{abstract}
A smart electrical car park with electric vehicles (EVs) parking there, regarded as a short-term storage system, could minimize the costs of EV customers and improve the main grid stability simultaneously. This system, including numerous bidirectional AC/DC converters, a local energy storage unit and a monitoring room, is firstly established. As the hourly prices of electricity fluctuating with time, EV owners would like to charge energy from the main grid during the low-price periods to save money, while discharging energy to the main grid during high-price periods. In order to achieve this, an optimal charging scheme is proposed to determine the charging rate of each $\mathrm{EV}$ based on the fluctuation of hourly prices and requirements of customers. Thus, this charging/discharging strategy can reduce the costs for EV owners and help keep the balance of supply and demand for the main grid. A comparison between the cost of EVs customers with and without the developed smart charging/discharging strategy in this smart electrical car park is presented and analysed in Matlab with an optimization problem solver named Cplex in this study. It is demonstrated that the proposed charging/discharging strategy can not only reduce EV owner's cost but also improve the main grid stability as well.
\end{abstract}

\section{Introduction}

The problem that energy sources, such as fossil fuels, are gradually used up, has attracted extensive attention all over the world recent years. Besides this, greenhouse gas emissions of traditional vehicles are also serious environmental problems that need to be solved $[1,2]$. Both of these problems promote the development and utilization of clean energy and renewable energy, which are economically and environmentally friendly. One positive and effective solution for these problems is using electric vehicles (EVs) to replace the traditional vehicles, such as fuel vehicles. Besides of renewable and environmental advantage, EVs can help keep the balance of supply and demand for the main grid [3].

With the increasing number of EVs, a car park with a large number of EVs parking there can be regarded as an energy store system. Since most vehicles spend $5 \%$ of the time on the road, while parking an average of $95 \%$ of the day [4], their batteries could be used to provide electricity flow from electric vehicles to the power lines and back. An EV can be charged during the peak-off hours, which is known as grid to vehicles $(\mathrm{G} 2 \mathrm{~V})$ [5]. Also, it could provide the energy stored in batteries to the main grid during the peak hours, which is known as vehicles to grid (V2G). When there are a lot of electrical vehicles (EVs) parking in the car park, these energies stored in vehicles' batteries will be very huge, which will play an important role in helping keep the balance of supply and demand in the grid [5-7].

Optimal charging algorithms have been studied in recent years [8-12], however, most of which focused on home charging or public charging. Some of them aimed to maximum the income of charger without considering profits of EV customers $[13,14]$. Even some of them take profits of EV customers into consideration [15]. However, the hourly electricity price and the demand of main grid are ignored. In order to maximize the benefits for the main grid and minimize the costs for EVs' owners, we developed a smart electrical car park model and proposed an optimizing charging/discharging scheme in this paper.

Compared with a traditional car park, a smart electrical car park can not only offer a parking spot for EVs but also charge/discharge EVs more economically. The proposed charging/discharging scheme can choose an appropriate charging power rate for each EV during the parking hours according to the hourly prices of electricity. In order to extend the life of batteries, the states of charge (SOCs) are set between $20 \%$ and $90 \%$ [3], except the demands of customers are beyond the range. At the end of parking time, EVs can be charged/discharged to the desired SOCs [16]. Also, the smart electrical car park allows EV customers to set their own demands: the final SOC, the departure time, maximum charging/discharge power rates, etc. In this paper, two charging methods are considered: a normal charging and an optimal charging. The normal charging is to charge or discharge the EV to the desired SOC when it comes in ignoring the electricity of price. The optimal charging is to find the best charging or discharging power and charging or discharging periods to reduce the costs of electricity for customers. Then, compared these two costs, it can be found that the proposed optimal charging scheme can help customers get the maximum profit and do a favour for the 
grid to keep the balance. We summarize our contributions as follows:

(1) We develop a model of smart electrical car park, which includes functions of a tradition car park, a monitoring room, chargers and energy storage devices;

(2) We present an optimal charging/discharging strategy for EVs to reduce the costs of customers and help the main grid keep a balance between supply and demand;

(3) We also compare costs with charging/discharging scheme to costs without any optimal algorithm, which is developed based on the survey of a University of Technology Sydney (UTS) car park.

\section{System model of Smart Electrical Car Parks}

In this section, a model of smart electrical car park is developed for EVs parking and charging/discharging, with main technology of G2V and V2G.

A smart electrical car park (SECP) is composed of a traditional car park, a monitoring room, several charging and discharging spots, and energy storage devices, as shown in Fig. 1. In such a system, the monitoring room serves as a central control node which collects information from both the main grid and EVs via WIFI and determines the charge or discharge rate of each EV during each period calculated by the smart charging algorithm. During the opening hours, the whole opening hours are divided into several periods and single decimal number between 0 and 24 is used to present a time. For example, suppose that the opening hours of a car park is from 7 a.m. to 22 p.m., and it will be divided into 30 periods with each period of half an hour. Then, the system state can be presented as $(7,22,30,0.5)$. Based on this, the charging rates will be updated every period by the charging algorithm and the charging states keep constant during each period. The key technology for such an electrical car park is V2G and G2V.

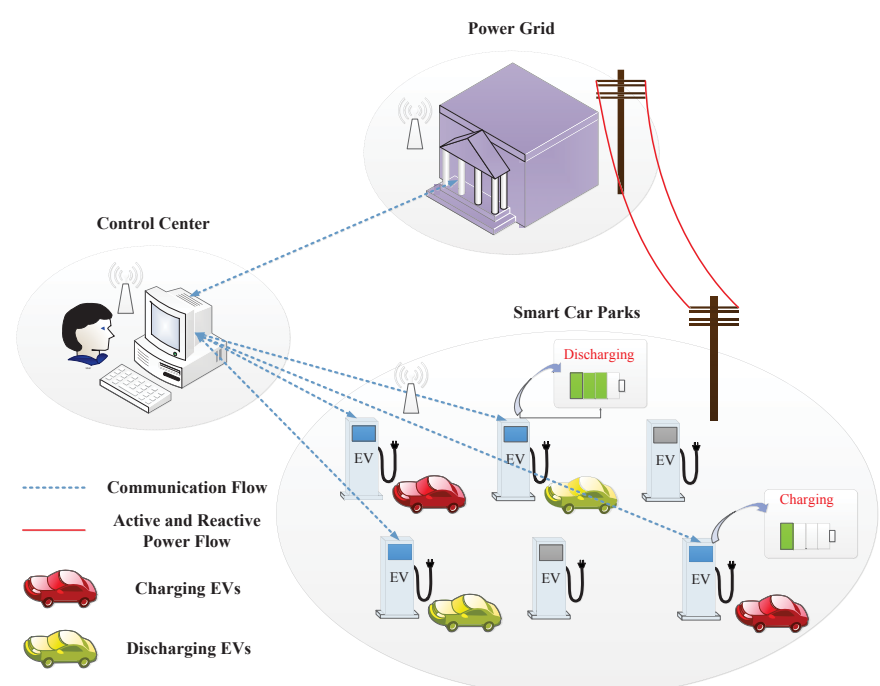

Fig. 1. Block diagram of SECP

Compared with other conventional car parks, SECP has several excellent economic advantages. It presents the advantages as followed:
(1) High utilization rate of the batteries which is used as storage devices when parking;

(2) Good assistant function for distributed power grid in power quality and power regulation by charging during valley period and discharging during peak period using the storage battery of EVs;

(3) Gain more economic profit for both car park carriers and EV owners.

Having such good characteristics, it makes SECP suitable for the future, especially when EVs play an important role in transportation.

\section{Proposed charging/discharging scheme}

\subsection{Charging powers of EVs}

Depending on the type of EV's battery and demand of the customer, each EV is able to be charged/discharged at any charging rate, ranging from a minimum value to a maximum one. In order to determine the ranges of charging power of EVs, EVs coming into the car park are divided into three categories according to its initial $\mathrm{SOC}\left(S O C_{\mathrm{m}, \mathrm{ni} i}\right)$, final $\mathrm{SOC}$ $\left(S O C_{\mathrm{m}, \text { fin }}\right)$, charging type and parking time. The initial $\mathrm{SOC}$ can be calculated when the EV plugging into the charger and the final SOC, charging type and parking time can be set by car owners through the interface on the charger.

3.1.1. Charging type

Charging type is set by car owners on the interface of the charger when parking cars. For simplicity, a parameter $R$ is used to represent the charging type. There are three states of $R$, which are:

(1) $R=1$ means the EV can only be charged during the parking time,

(2) $R=0$ means the EV can be charged and discharged during the parking time,

(3) $R=-1$ means the EV can only be discharged during the parking time.

3.1.2. Parking time

These groups can be divided into three subtypes according to the maximum idle parking time, which can be calculated by the following equation:

$$
t_{\mathrm{m}, \mathrm{p}, \max }=t_{\mathrm{m}, \mathrm{p}}-t_{\mathrm{m}, \min , c}
$$

where $t_{\mathrm{m}, \mathrm{p} \text {,max }}$ is the maximum idle parking time for EV $m$ th, $t_{\mathrm{m}, \mathrm{p}}$ the total parking time and $t_{\mathrm{m}, \mathrm{min}, \mathrm{c}}$ the minimum charging or discharging time. $t_{\mathrm{m}, \mathrm{p}}$ is the parking time from EV $m$ th plugged in to the charger drawn out.

3.1.3. Charging power

The maximum charging or discharging powers, denoted by $p_{\mathrm{m}, \mathrm{c}, \max }$ and $p_{\mathrm{m}, \mathrm{d}, \max }$, respectively, should be also be set by customers or calculated by chargers.

We define that the charging power of EV $m$ th is positive and the discharging power is negative, which can be given as

$$
\begin{aligned}
& p_{m, c, \text { max }}>0 \\
& p_{m, d, \text { max }}<0
\end{aligned}
$$


Based on the statement of classification of EVs, power ranges of EVs can be determined when coming into the car park (shown in Table 1). Two cases are impossible for EVs: (1) One is that consumers need the final SOC is larger than initial one with the type $R=-1$ (can only be discharging), (2) Another one is that consumers need the final SOC is lower than initial one with the type $R=1$ (can only be charging). These two impossible cases are defined as error cases. In Table 1, $\Delta t$ is a time slot (h) and $p_{\mathrm{m}}$ is the charging or discharging power of EV $m$ th $(\mathrm{kW})$.

Table 1: Charging power for EVs.

\begin{tabular}{|c|c|c|c|}
\hline \multirow{2}{*}{ The state of SOC } & \multirow{2}{*}{$\begin{array}{c}\text { Charging } \\
\text { type }\end{array}$} & \multicolumn{2}{|c|}{ The charging power } \\
\hline & & $t_{\mathrm{m} \mathfrak{n} \max } \leq \Delta \mathrm{t}$ & $t_{\text {mp max }}>\Delta \mathrm{t}$ \\
\hline \multirow{3}{*}{$S O C_{\mathrm{m}, \mathrm{ini}}>S O C_{\mathrm{m}, \mathrm{fin}}$} & $R=1$ & \multirow{3}{*}{$p_{\mathrm{m}}=p_{\mathrm{m}, \mathrm{c}, \max }$} & $p_{\mathrm{m}} \in\left[0, p_{\mathrm{m}, \mathrm{c}, \max }\right]$ \\
\hline & $R=0$ & & $p_{\mathrm{m}} \in\left[p_{\mathrm{m}, \mathrm{d}, \max }, p_{\mathrm{m}, \mathrm{c}, \max }\right]$ \\
\hline & $R=-1$ & & Error \\
\hline \multirow{3}{*}{$S O C_{\mathrm{m}, \text { ini }}=S O C_{\mathrm{m}, \text { fin }}$} & $R=1$ & \multirow{3}{*}{$p_{\mathrm{m}}=0$} & $p_{\mathrm{m}}=0$ \\
\hline & $R=0$ & & $p_{\mathrm{m}} \in\left[p_{\mathrm{m}, \mathrm{d}, \max }, p_{\mathrm{m}, \mathrm{c}, \max }\right]$ \\
\hline & $\begin{array}{l}R=-1 \\
R=1\end{array}$ & & $\begin{array}{c}p_{\mathrm{m}}=0 \\
\text { Error }\end{array}$ \\
\hline \multirow[t]{2}{*}{$S O C_{\mathrm{m}, \mathrm{ini}}<S O C_{\mathrm{m}, \mathrm{fin}}$} & $R=0$ & \multirow[t]{2}{*}{$p_{\mathrm{m}}=p_{\mathrm{m}, \mathrm{d}, \max }$} & $p_{\mathrm{m}} \in\left[p_{\mathrm{m}, \mathrm{d}, \max }, p_{\mathrm{m}, \mathrm{c}, \max }\right]$ \\
\hline & $R=-1$ & & $p_{\mathrm{m}} \in\left[p_{\mathrm{m}, \mathrm{d} \max }, 0\right]$ \\
\hline
\end{tabular}

\subsection{Parking fee}

The cost of parking fee $c_{\mathrm{m}, \mathrm{PF}}$ is the traditional parking fee of each car for using the spot. It is related with parking hours and parking fee per hour.

\subsubsection{Parking hours}

The parking hours for EV $m$ th $\left(P H_{\mathrm{m}}\right)$ is related to parking time $t_{\mathrm{m}, \mathrm{p}}$. When $t_{\mathrm{m}, \mathrm{p}}$ is not equal to integer times of hours, parking hours for each EV can be calculated by the following equation:

$$
P H_{m}=\left[t_{m, p}\right]+1
$$

where $P H_{\mathrm{m}}$ is minimum number of parking hours.

Otherwise, the parking hours can be calculated by:

$$
P H_{m}=t_{m, p}
$$

\subsubsection{Parking fee}

Then, the parking fee $c_{\mathrm{m}, \mathrm{PF}}$ can be calculated as:

$$
c_{m, P F}=P H_{m} \times k_{m}
$$

where $k_{\mathrm{m}}$ is the parking price per hour $(\mathrm{A} \$ / \mathrm{h})$.

\subsection{Constraints}

\subsubsection{Parking time}

EVs should be parking during the opening hours of the car park, which means that the starting time should be later than the opening time and the ending time should be earlier than the closing time.

\subsubsection{Charging power}

The powers should be limited by maximum charging or discharging powers during the charging mode and discharging mode, respectively, which are given by:

Charging mode: $0 \leq p_{m} \leq p_{m, c, \max }$

Discharging mode: $p_{m, d, \max } \leq p_{m} \leq 0$

\subsubsection{Range of SOC}

In order to extend battery lifetime, the final SOC is set as the default range between $20 \%$ and $90 \%$ by the car park if car owners do not set it. However, if the final SOC is set out of this range, the $\mathrm{EV}$ could be charged or discharged to the desired value.

3.3.4. Energy demand

The needed energy of EV $m$ th, $E_{\mathrm{m}, \mathrm{n}}$, can be calculated as:

$$
E_{m, n}=C_{m} \times\left(S O C_{m, f i n}-S O C_{m, i n i}\right)
$$

where $C_{\mathrm{m}}$ is the battery capacity $(\mathrm{kWh})$.

If $\mathrm{EV} m$ th has enough time to be charged or discharged, the total charging or discharging energy would be equal to the needed energy, which is given as:

$$
E_{m, n}=\sum_{n=1}^{N_{m}} E_{m}(n)=\sum_{n=1}^{N_{m}}\left(p_{m, n} \times \Delta t\right)
$$

where $E_{\mathrm{m}}(\mathrm{n})$ is the charging or discharging energy of EV $m$ th during $n$th period $\Delta t$ and $N_{\mathrm{m}}$ the total number of periods during the parking time for EV $m$ th, which can be calculated by:

$$
N_{m}=\left[\frac{t_{m, p}}{\Delta t}\right]
$$

The charged energy of EV $m$ th during any periods, calculated by Equation (12), should be no less than 0 and no larger than the needed energy, as shown in Equation (13):

$$
\begin{gathered}
E_{m}=S O C_{m, i n i} \times C_{m}+\sum_{n=1}^{N_{m}{ }^{\prime}} E_{m}(n) \quad N_{m}{ }^{\prime} \in\left[0,\left[\frac{t_{m}}{\Delta t}\right]\right] \\
0 \leq E_{m} \leq E_{m, n} \leq C_{m}
\end{gathered}
$$

\subsection{Cost function}

The electricity price is a function of time, which can be denoted as price $=f(t)$. The price is assumed to be constant during each time slot $\Delta t$ and the charging price, $P_{\mathrm{c}}$, is equal to the discharging price, $P_{\mathrm{d}}$, which is $P_{\mathrm{c}}=P_{\mathrm{d}}$.

For EV $m$ th, the cost of charging and discharging electricity are shown as:

$$
\begin{aligned}
& c_{m, c h g}=\sum_{n=1}^{n=N_{m}}\left(P_{c}(n) \times E_{m}(n)\right) \\
& c_{m, \text { dis }}=\sum_{n=1}^{n=N_{m}}\left(P_{d}(n) \times E_{m}(n)\right)
\end{aligned}
$$

It is obviously that the cost of discharging electricity is lower than 0 . Therefore, the total cost for EV $m$ th car owner can be calculated by:

$$
c_{m, t}=c_{m, c h g}+c_{m, P F}+c_{m, d i s}
$$

Then the objective of the proposed scheme is minimizing the cost for customers, which can be recorded as:

$$
\text { objective }=\min \left(c_{m, t}\right)=\min \left(c_{m, c h g}+c_{m, P F}+c_{m, \text { dis }}\right)
$$

\subsection{Algorithm}

Matlab routes the optimization model to the solver-IBM ILOG CPLEX Optimizer 12.6.1 to solve the optimization 
problem, as shown in Fig. 2 and Table 2. Yalmip Wiki is a tool employed to build the mathematical model in Matlab.

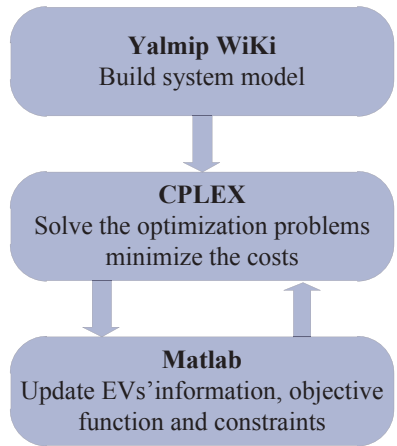

Fig. 2. Framework of the proposed charging scheme.

Table 2: Main Algorithm.

\begin{tabular}{|c|c|}
\hline \multicolumn{2}{|r|}{ Main Algorithm } \\
\hline Step: & $\%($ get the basic information of each EV) \\
\hline 1 & EV=[ 'NO','Cm','SOCin','SOCfin','R','Pcmax','Pdmax','tc ,'te ] \\
\hline & \%Solve the problem by using Cplex \\
\hline & for $\mathrm{i}=1: \mathrm{M}$ \\
\hline & for $\mathrm{j}=1: \mathrm{N}$ \\
\hline & Constraints $=$ \\
\hline & {$[$ Constraints, $20 \% * \mathrm{Cm}<=$ energy $<=90 \% * \mathrm{Cm}, \ldots]$} \\
\hline & $\operatorname{Pm}(\mathrm{i}, 2)<=\mathrm{p}(1, \mathrm{j})<=\operatorname{Pm}(\mathrm{i}, 1), \operatorname{sum}(\mathrm{x}(1,:), 2) * \mathrm{dt}==\mathrm{En}(\mathrm{i}, 1)]$ \\
\hline Step: & $\mathrm{c}=$ Price. ${ }^{*} \mathrm{p}(1, ;)^{*} \mathrm{dt}$ \\
\hline 2 & Objective $=\min (\operatorname{sum}(c, 2))$ \\
\hline & $\%$ Set some options for YALMIP and solver \\
\hline & 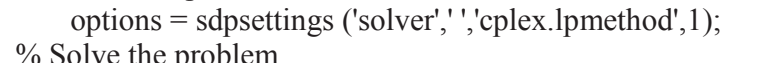 \\
\hline & $\begin{array}{l}\text { sol = optimize(Constraints, Objective, options); } \\
\text { end }\end{array}$ \\
\hline & end \\
\hline $\begin{array}{l}\text { Step: } \\
3\end{array}$ & \% Show each EV's charging power during each time slot \\
\hline
\end{tabular}

\section{Simulation and results}

In this section, a car park, located in Building 10 in University of Technology Sydney (UTS), with 150 parking positions, is selected as a research object, whose opening hours are from 7 a.m. to 22 p.m. during Monday to Friday.

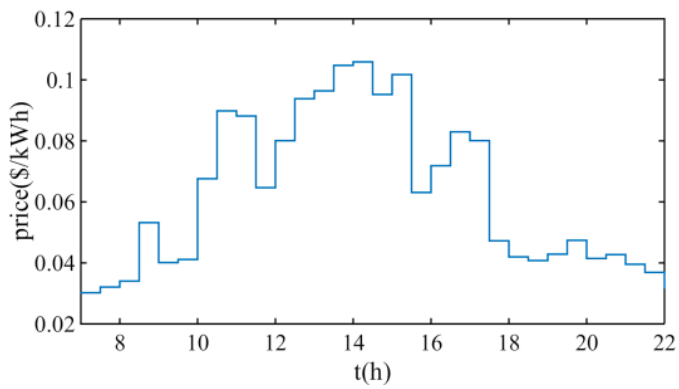

Fig. 3. Average hourly electricity prices in NSW.

The performance of the proposed charging algorithm is evaluated by employing real hourly electricity prices, EV battery data, a real car park and parking information about cars. Specifically, the hourly electricity prices in NSW on each day (January 1 January 30, 2015) were obtained from AEMO (Australian Energy Market Operator) [17], which operates the National Electricity Market (NEM) of south eastern Australia. Average hourly electricity prices over 30 days are used for simulation during 7 a.m. to 22 p.m., as shown in Fig. 3.

\subsection{Distribution of EVs}

In order to develop an accurate car park system model, the probability density function of EVs parking information is needed. A survey on this UTS car park has been done during a whole week from $19 / 1 / 2015$ to $25 / 1 / 2015$.

Based on this investigation, the information of the parking time, the arriving time and the departure time of each car can be obtained. The probability density function of parking duration time is a normally distributed random variable with $\mu=8.299$ and $\sigma=1.814$, as shown in Fig. 4. The distribution function for parking hours is given by:

$$
f(x ; \mu, \sigma)=\frac{1}{\sqrt{2 \pi} \sigma} \exp \left\{-\frac{(x-\mu)^{2}}{2 \sigma^{2}}\right\}
$$

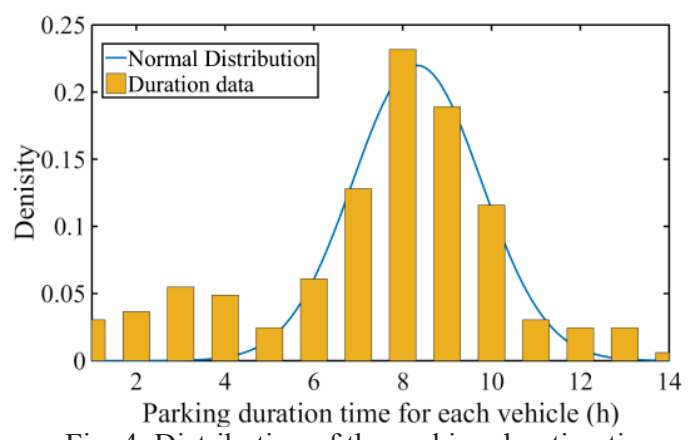

Fig. 4. Distribution of the parking duration time.

Arriving and departure times are also normal probability density functions. The distribution parameters of arriving and departure times are shown in Table 3 . The probability density functions of them are shown in Fig. 5 and 6, respectively.

Table 3: Distribution parameters of arriving and departure times.

\begin{tabular}{|c|c|c|}
\hline Parameter & Arrival & Departure \\
\hline$\mu$ & 9.021 & 17.22 \\
$\sigma$ & 0.8755 & 1.7572 \\
\hline
\end{tabular}

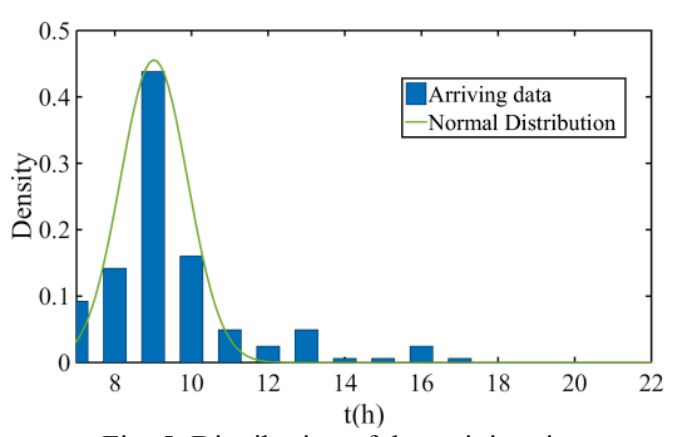

Fig. 5. Distribution of the arriving time. 


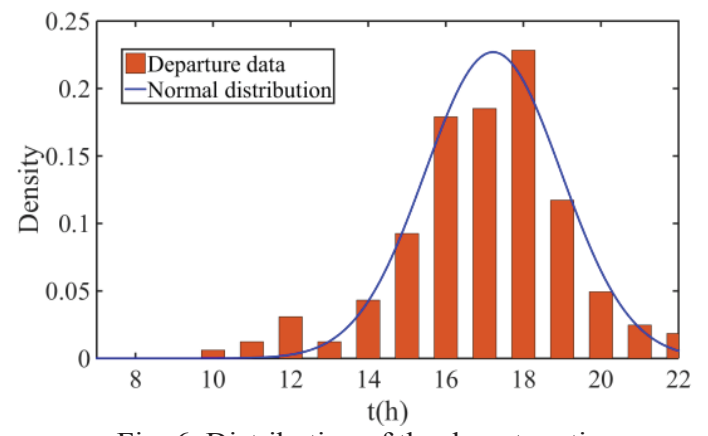

Fig. 6. Distribution of the departure time.

Although these cars are traditional vehicles, it is reasonable to assume that the behaviours for drivers would change little in the future. Therefore, the information from the survey is able to be utilized in the simulation setting.

\subsection{Simulation result}

In the simulation, initial SOCs of EVs are set from $10 \%$ to $90 \%$, final SOCs are set from $50 \%$ to $90 \%\left(S O C_{\mathrm{m}, \text { fin }}\right.$ is set by customers) and capacity of EVs is from $30 \mathrm{kWh}$ to $100 \mathrm{kWh}$ (this is according to the behaviour of drivers). The proposed charging scheme is set to update the information of EVs every $30 \mathrm{~min}$. All the simulation settings are shown in Table 4. M is the total number of EVs parking in this smart electrical during one day. The distributions of arriving, departure times and parking hours of EVs are selected according to the normal probability density functions mentioned before.

Table 4: Simulation settings.
\begin{tabular}{|c|c|}
\hline Parameters & Values \\
\hline$T_{\text {open }}$ & 7 a.m. \\
$T_{\text {close }}$ & 22 p.m. \\
$T_{\mathrm{m}, \mathrm{s}}$ & 7 a.m. $\sim 22$ p.m. \\
$T_{\mathrm{m}, \mathrm{e}}$ & 7 a.m. $\sim 22$ p.m. \\
$C_{\mathrm{m}}$ & $30 \sim 100 \mathrm{kWh}$ \\
$S O C_{\mathrm{m}, \text { ini }}$ & $10 \% \sim 90 \%$ \\
$S O C_{\mathrm{m}, \mathrm{fin}}$ & $50 \% \sim 90 \%$ \\
$\Delta t$ & $0.5 \mathrm{~h}$ \\
$M$ & 100 \\
\hline
\end{tabular}

Fig. 7 shows the final SOCs of $100 \mathrm{EVs}$ after parking in the car park and the expected values set by car owners. It can be seen that the final SOC of EV 56th is lower than the desired value. This because it only parks only one hour that it cannot be charged to the desired SOC even with the maximum charging rate. Except EV 56th, all the EVs can meet the demands set by car owners.

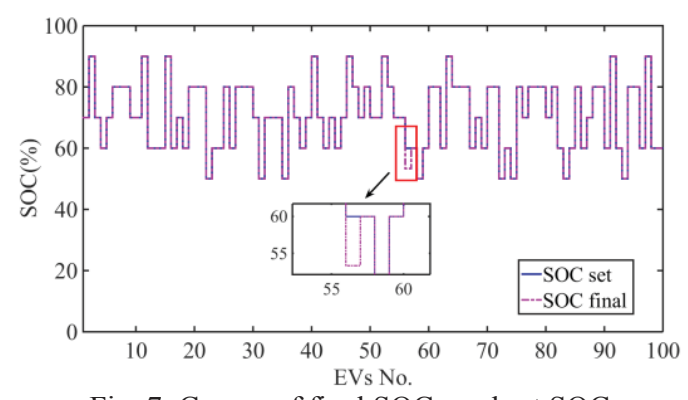

Fig. 7. Curves of final SOCs and set SOCs.
Three EVs (EV 10th, EV 15th and EV 35th) are chosen randomly to observe their SOCs. The variation of their SOCs and hourly prices of electricity are depicted in Fig. 8. It can be seen that they can be charged during the low-price periods and be discharged during the high-price periods.

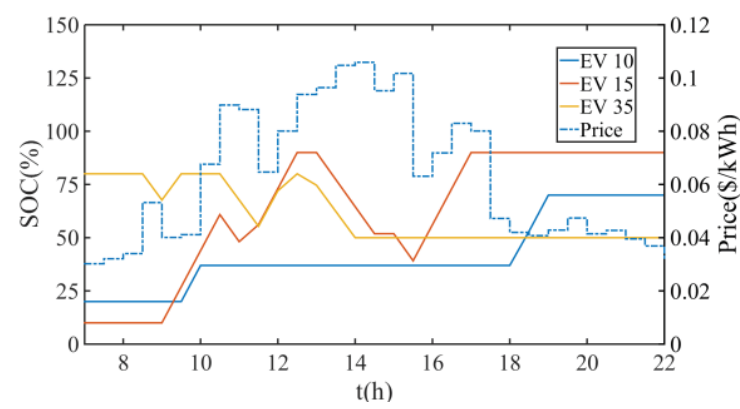

Fig. 8. Three EVs' SOCs and hourly electricity prices.

The simulation results with the proposed and the normal charging scheme are also compared in this paper. The difference between the costs with and without the proposed charging algorithms is shown in Fig. 9. For all the EVs, the costs with proposed charging algorithm are less than or equal to the cost with the normal charging algorithm.

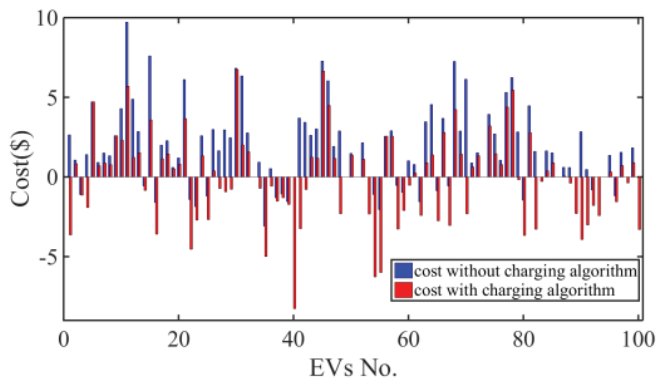

Fig. 9. Two costs by two charging methods.

From Fig. 10, it shows that the saving money for 100 EVs. Each EV would save 3 Australian dollars on average during a whole day. Some EVs discharging electricity to the main grid can also earn money.

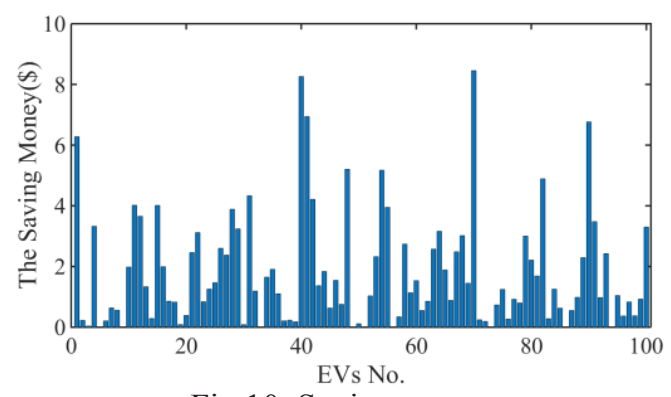

Fig.10. Saving money.

\subsection{Improve power grid stability}

The relationship between total demand and average regional reference price on one day is shown in Fig. 11, which obtained from AEMO during a whole month. It can be seen that the price is higher when the demand is large. This means that if EVs discharge energy to the main grid during the highprice hours/peak hours, it will reduce the electricity stress for 
the grid. It will help grid to gain more demand during the offpeak hours, vice versa, which helps keep the balance between the demand and supply for the main grid.

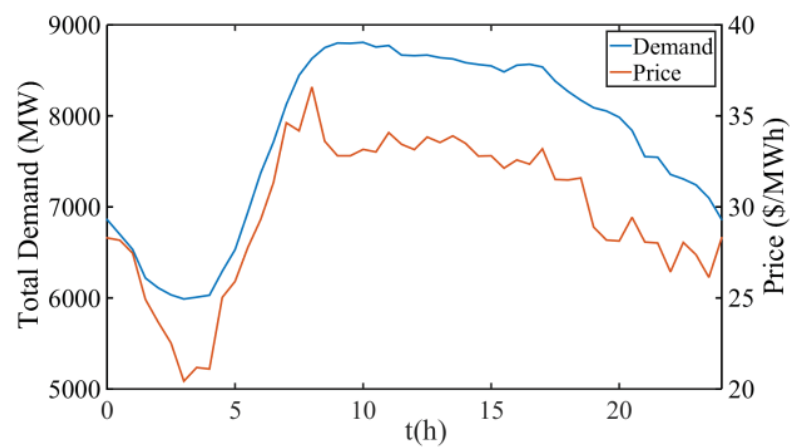

Fig. 11. Electricity demand and average regional reference price.

\section{Conclusion}

In this paper, a smart electrical car park model has been proposed and an optimal charging scheme has been studied by considering the hourly electricity prices to maximize the profit for EVs owners. The proposed charging scheme is applied in UTS official car park with 100 parking slots. Compared the costs with and without the proposed charging algorithms, the simulation results show that the proposed charging scheme can help EV owners save money and generate charging/discharging power rates more economically. Moreover, it can help the main grid peak load shifting as well. For EV owners, it is obvious that they would prefer to park their cars in such a smart electrical car park, which can save their money. This will help car park owners enlarge their parking market and gain more benefits from parking service.

\section{Acknowledgements}

The authors gratefully acknowledge the support for this research from the China Scholarship Council (CSC) (201407090060) and University of Technology Sydney.

\section{References}

[1] Zhang L, Jabbari F, Brown T. "Coordinating plug-in electric vehicle charging with electric grid: Valley filling and target load following", Journal of Power Sources, 267, pp. 584-597, (2014).

[2] García-Villalobos J, Zamora I, San Martín J I. "Plug-in electric vehicles in electric distribution networks: A review of smart charging approaches", Renewable and Sustainable Energy Reviews, 38, pp. 717-731, (2014).

[3] Vo T T, Chen X, Shen W. "New charging strategy for lithium-ion batteries based on the integration of Taguchi method and state of charge estimation", Journal of Power Sources, 273, pp. 413-422, (2015).

[4] Zhang L, Brown T, Samuelsen S. "Evaluation of charging infrastructure requirements and operating costs for plug-in electric vehicles", Journal of Power Sources, 240, pp. 515-524, (2013).
[5] Habib S, Kamran M, Rashid U. "Impact analysis of vehicle-to-grid technology and charging strategies of electric vehicles on distribution networks-A review", Journal of Power Sources, 277, pp. 205-214, (2015).

[6] Su W, Chow M Y. "Computational intelligence-based energy management for a large-scale PHEV/PEV enabled municipal parking deck", Applied Energy, 96, pp. 171-182, (2012).

[7] Kennel F, Gorges D, Liu S. "Energy management for smart grids with electric vehicles based on hierarchical MPC", IEEE Transactions on Industrial Informatics, 9, pp. 1528-1537, (2013).

[8] Ahn C, Li C T, Peng H. "Optimal decentralized charging control algorithm for electrified vehicles connected to smart grid", Journal of Power Sources, 196, pp. 10369-10379, (2011).

[9] Nguyen H K, Song J B. "Optimal charging and discharging for multiple PHEVs with demand side management in vehicle-to-building", Journal of Communications and Networks, 14, pp. 662-671, (2012).

[10] He Y, Venkatesh B, Guan L. "Optimal scheduling for charging and discharging of electric vehicles", IEEE Transactions on Smart Grid, 3, pp. 1095-1105, (2012).

[11] Su W, Wang J, Zhang K. "Model predictive controlbased power dispatch for distribution system considering plug-in electric vehicle uncertainty", Electric Power Systems Research, 106, pp. 29-35, (2014).

[12] Bashash S, Fathy H K. "Cost-optimal charging of plugin hybrid electric vehicles under time-varying electricity price signals", IEEE Transactions on Intelligent Transportation Systems, 15, pp. 1958-1968, (2014).

[13] $\mathrm{Su}$ W, Chow M Y. "Performance evaluation of an EDAbased large-scale plug-in hybrid electric vehicle charging algorithm", IEEE Transactions on Smart Grid, 3, pp. 308-315, (2012).

[14] Jin C, Tang J, Ghosh P. "Optimizing electric vehicle charging: a customer's perspective", IEEE Transactions on Vehicular Technology, 62, pp. 2919-2927, (2013).

[15] Neubauer J, Wood E. "The impact of range anxiety and home, workplace, and public charging infrastructure on simulated battery electric vehicle lifetime utility", Journal of Power Sources, 257, pp. 12-20, (2014).

[16] Khayyam H, Ranjbarzadeh H, Marano V. "Intelligent control of vehicle to grid power", Journal of Power Sources, 201, pp. 1-9, (2012).

[17] Australian Energy Market Operator (AEMO), Electricity Data, 2015. Available from: http://www.aemo.com.au/. 